\title{
Variables Associated with Alcohol Consumption and Abstinence among Young Adults in Central China
}

\author{
Ling Qian ${ }^{1}$, Ian M. Newman ${ }^{2, *} \mathbb{1}$, Lok-wa Yuen ${ }^{2}$, Duane F. Shell ${ }^{2}$ and Jingdong $\mathrm{Xu}^{3}$ \\ 1 Chinese Center for Health Education (CCHE), Beijing 100011, China; qianlingzh@126.com \\ 2 Department of Educational Psychology, University of Nebraska-Lincoln, Lincoln, NE 68588, USA; \\ lok-wa.yuen@huskers.unl.edu (L.-w.Y.); dshell2@unl.edu (D.F.S.) \\ 3 Hubei Institute for Health Education, Wuhan 430079, China; hbsxjd@163.com \\ * Correspondence: inewman1@unl.edu; Tel.: +1-402-304-4036
}

Received: 18 May 2018; Accepted: 4 August 2018; Published: 7 August 2018

\begin{abstract}
This paper presents a descriptive analysis of data gathered by personal interviews from a multistage random sample of 1640 residents aged 18-34 years in Wuhan, China. First, alcohol drinkers and abstainers were compared based on demographic, attitude, and belief variables. Next, the drinkers from the sample were classified into four groups based on frequency-quantity of alcohol use, and the frequency-quantity groups were compared on the same variables. For Abstainers versus Drinkers, we found no difference by age or gender in this sample. Married people and people with children were more likely to be abstainers. University-educated, currently-employed individuals in mid-level jobs were more likely to abstain from alcohol. Vocational/Technical graduates, people who were currently attending college, currently unemployed and never-employed individuals were more likely to be drinkers. Abstainers also responded with less-positive attitudes and beliefs about drinking and attached more importance to reasons for not drinking compared to drinkers. When the drinking frequency-quantity groups were compared, gender differences became significant: more high-quantity drinkers were women; however the guideline for quantity for women was $>1$ drink at a time compared to $>2$ drinks at a time for men. Quantity and frequency of drinking was significantly associated with having children, educational level, employment status, and type of occupation. Age, marital status, and being in college did not relate significantly with quantity and frequency of drinking alcohol. Attitudes and beliefs about drinking tended to be more positive among high-frequency and high-quantity drinkers. Drinkers in all frequency-quantity groups attached greater importance to social reasons for drinking compared to personal/psychological reasons for drinking. Drinkers in the lowest frequency-quantity group attached the most importance to reasons for not drinking. These findings confirmed that in China drinking plays an important role in socializing and celebrating, and that there are important differences between alcohol drinkers and abstainers and between frequency/quantity groups of drinkers. Western models of individualized motivation of behaviors may not accurately explain alcohol use in China. We believe the findings from this study suggest the need for more detailed studies of alcohol drinking and abstaining.
\end{abstract}

Keywords: alcohol patterns; survey; interview; frequency; quantity; abstaining; heavy drinking; high-risk drinking

\section{Introduction}

There is limited data available on the drinking patterns of specific age groups in China. The available literature has described the socio-demographic characteristics of alcohol use [1-5], attitudes toward alcohol drinking [5-7], drinking motives [1,8,9], family factors related to drinking [1], 
peer alcohol use [1,5], drinking situations [1,7], health status [6,10], other substance use $[2,5,6]$, and mental health problems [11,12]. Little attention has been paid to young adults as a group.

Descriptions of alcohol use among young people have typically been limited to student populations, and have overlooked young adults in general $[13,14]$. This is the first known attempt to describe the drinking characteristics of young Chinese adults ages 18 to 34 years. The data for this analysis were originally gathered as part of a four-country alcohol consumption patterns and behaviors study among young adults aged 18 to 34 by Taylor et al. [15]. Taylor et al.'s results illustrated the major differences in alcohol consumption patterns among young adults in Nigeria, Uruguay, Russia, and China. For example, the proportion of young adults who "ever drank" alcohol was 33.3\% (Ilorin, Nigeria), 53.4\% (Wuhan, China), 86.1\% (Moscow, Russia), and 96.4\% (Montevideo, Uruguay); the proportion of the sample who were abstainers was $79.8 \%, 54.2 \%, 23.1 \%$, and $12.3 \%$ respectively. There is a need to look at the individual country sample data more closely. This paper presents a detailed analysis of the data from the China sample to see what we can learn about alcohol use and abstinence among 18-34 year olds. The results are presented in two parts. Part 1 describes the differences and similarities between drinkers and abstainers, and Part 2 explores the differences among current drinkers based on the quantity and frequency of drinking. These results will be of interest to international readers who are interested in alcohol survey methods and questions for use in China studies, and for those interested in the development of nuanced policy and education to reduce alcohol consumption. China's population represents $19 \%$ percent of the world's population and deserves careful attention so that proposed international alcohol policies might be effective in regions of the world outside the EU and USA.

In 2010 , in China, it was estimated that $58.4 \%$ of the males and $28.9 \%$ of the females, aged 15 years and older had consumed alcohol in the past 12 months [16]. Among these drinkers the estimated per capita consumption for males aged 15 and older was $18.7 \mathrm{~L}$ of pure alcohol and for females 7.6 L [16]. The prevalence of personal alcohol-related, medically-defined conditions among males was estimated to be $4.4 \%$ for alcohol dependence, $4.0 \%$ for alcohol abuse, and $10.1 \%$ for alcohol use disorder [11]. The risk of developing AUD peaked at age 30 [12].

Chinese alcohol culture shares many common characteristics with other alcohol-consuming cultures, but it also differs in important ways. Chinese traditionally view alcohol drinking as an important tool for social networking and emotional expression. Business meetings, social events, and special celebrations like weddings, funerals, and festivals are often accompanied by drinking alcohol [17]. Moderate drinking is common. Nevertheless, certain drinking customs create significant risks. For example gatherings of friends and colleagues often involve urging others to drink to establish rapport and confirm relationships $[17,18]$. Toasting is a way to show respect to others. Guests usually feel obligated to drink as much as the host demands. These culturally-respected social pressures make refusing to drink very difficult. In work situations younger employees are sometimes asked to drink for their supervisors and other administrators. Being capable of drinking a lot is seen as a networking ability [1], however acting badly as a result of drinking is a cause for shame [19].

\section{Materials and Methods}

\subsection{The Data}

The data used in this secondary analysis were derived from a multistage random sample of residents aged 18-34 in Wuhan, China. Wuhan is composed of 13 administrative districts. The sample size for each administrative district was proportional to the population size. Within each administrative district, communities were randomly selected, followed by random household selection. A household member aged 18-34 who had lived in the city for at least 6 months was eligible for interview. If more than one household member was eligible, the person with the most recent birthday was selected. No replacement was allowed for non-respondents. A sample size of 1600 was planned; 1640 interviews were completed [15]. 
To reduce potential bias and ensure the results accurately reflected the population of interest, the data were weighted by administrative district (urban vs. sub-urban area), age (18 to 34 years), gender, and probability of selection in the household.

\subsection{The Survey Instrument}

The survey instrument was developed by a multinational team and collected information about alcohol use, drinking effects, drinking motives, and perception of alcohol drinking. The questionnaire used in China was developed in English, forward translated to Chinese and back translated to English. Modifications accommodated cultural and linguistic nuances. It was pretested with a small sample in Wuhan and further refined. The final questionnaire was administered in face-to-face interviews conducted by specially trained epidemiology graduate students. Interviews lasted about $15 \mathrm{~min}$ and were conducted in environments where respondents felt comfortable.

\subsection{Classification of Drinkers, Abstainers, and Drinking Frequency-Quantity Groups}

\subsubsection{Drinkers and Abstainers}

Respondents were divided into drinkers and abstainers based on their answers to three alcohol consumption questions: (1) had ever consumed alcohol (excluding sips), (2) during the last 12 months how often had consumed alcohol, even a small amount, and (3) during the past 12 months, how many drinks on a typical day. Drinkers $(n=704)$ were respondents who drank alcohol in the past 12 months and consumed at least one standard drink. Abstainers $(n=763)$ were respondents who have never drunk any alcohol. To distinguish clearly between drinkers and abstainers, respondents who had ever consumed alcohol but not in the last 12 months $(n=122)$ and respondents who consumed in the past 12 months but less than one standard drink $(n=47)$ were excluded from this study. These former drinkers and occasional drinkers were too few in number to justify inclusion, and the survey provided no information on their drinking histories. Four individuals who did not provide complete drinking information were also excluded from the study. The comparison of drinkers and abstainers is presented in Part 1.

\subsubsection{Drinking Frequency-Quantity Groups}

To understand more about the drinkers, they were further divided into groups based on frequency and quantity of drinking. Drinkers who drank at least once a month were labeled high-frequency drinkers and those who drank less than once a month were labeled low-frequency drinkers. Our quantity measure was based on Wang, Lay, Yu and Shen's [20] discussion of the Dietary Guidelines for Chinese Residents that defined moderate alcohol consumption for women as less than $15 \mathrm{~g}$ of pure alcohol per day and for men as less than $25 \mathrm{~g}$ per day. Since one standard drink contains $10 \mathrm{~g}$ of alcohol in this study, drinkers were considered high-quantity if they consumed more than two drinks at a time for males and more than one drink at a time for females.

Using these criteria, four drinking groups were created: low-frequency-low-quantity (LoF-LoQ) drink less than once a month and no more than the 1-2 drinks at a time as recommended for moderate alcohol consumption; high-frequency-low-quantity (HiF-LoQ) drink more than once a month but not more than the 1-2 drink guideline; low-frequency-high-quantity (LoF-HiQ) drink less than once a month but more than the recommended 1-2 drinks when they did drink; and high-frequency-high-quantity (HiF-HiQ) drink at least once a month and more than the 1-2 drinks recommended. Comparison among the four different drinking groups is presented in Part 2.

\subsection{Variables in Part 1}

Part 1 of this analysis compared drinkers and abstainers on four variables: demographics, perceptions of drinking, acceptance of drinking, and reasons for not drinking. 


\subsubsection{Demographic Information}

Demographic information included age, gender, marital status, highest education level attained, employment status, occupation, if a student or not, whether or not the interviewee had children.

\subsubsection{Perceptions of Drinking}

Perceptions of drinking were assessed by three items on a 5-point bipolar scale from strongly disagree to strongly agree. They were (1) drinking is one of life's pleasures, (2) drinking with someone else is a way of showing friendship, and (3) drinking has no benefits. With the value of skewness and excess kurtosis between -1 and +1 for all items, each item was considered as normally distributed and treated as a continuous variable, so a higher score indicated a more positive attitude towards alcohol. De Winter and Dodou [21] have concluded that a $t$-test and a Mann-Whitney-Wilcoxon test for five-point Likert items generally yielded the same results and had similar power.

\subsubsection{Acceptance of Drinking}

Acceptance of drinking was assessed in response to eight gender-specific drinking situations on a 5-point scale (should not drink, can drink a little but without any real effects, can drink and feel some effects but not get intoxicated, getting intoxicated is sometimes ok, and getting intoxicated is always ok). For measurement parsimony, principal component analysis, using orthogonal and oblique rotations, was conducted to create a smaller set of components. An oblique rotation provided the best defined 4-component structure with loadings above 0.3 (Table S1). The components were: (1) With Young Children ( 2 items: a mother spending time with young children, and a father spending time with young children), (2) Adult Private Context (2 items: a man having dinner with partner/spouse/cohabitant at home, and a woman having dinner with partner/spouse/cohabitant at home), (3) Social Drinking for Males (2 items: a man at a bar with friends, and a man out with colleagues), and (4) Social Drinking for Females ( 2 items: a woman out with colleagues, and a woman at a bar with friends). The components were statistically correlated ranging from -0.555 to 0.517 . Internal consistency was examined using Cronbach's alpha -0.871 for With Young Children, 0.927 for Adult Private Context, 0.777 for Social Drinking for Males, and 0.831 for Social Drinking for Females. Component scores were computed as the mean of the items in each of the component. A higher mean score indicated a greater acceptance of alcohol drinking.

\subsubsection{Reasons for Not Drinking}

Respondents were asked to rate the importance of 13 reasons for not drinking on a 4-point scale (not at all important, not very important, important, very important). A factor analysis failed to identify a coherent factor structure from the 13 scores, so cluster analysis was used to reduce the number of variables and identify membership groups. Three clusters emerged (Table S2). A low-rating group thought most of the reasons for not drinking were not at all important or unimportant, a high-rating group thought that most of the reasons for not drinking were important or very important, and a mid-range group whose scores were in between. The clusters suggested that respondents tended to rate all the reasons for not drinking as unimportant or all the reasons for not drinking as important. Only the mid-range cluster appeared to attribute different levels of importance to the different reasons for not drinking.

\subsection{Additional Variables in Part 2}

Part 2 of this analysis was among the four drinking frequency-quantity groups. In addition to demographic variables, perceptions of drinking, acceptance of drinking, and reasons for not drinking, comparisons were made on the effects of drinking as well as reasons for drinking. Alcohol use in different drinking locations/situations is presented as an introduction to Part 2. 


\subsubsection{Drinking Locations/Situations}

Drinkers were asked to identify in which of seven locations/situations they drank alcohol: (1) drinking in restaurants for dinner, (2) in restaurants for lunch, (3) in bars, (4) at friends' homes, (5) at their own home with friends, (6) at their own home alone, and (7) in public places like parks.

\subsubsection{Effects of Drinking}

Drinkers were asked to report on 13 possible effects alcohol had on them. Answers were on a 5-point scale (never, seldom, sometimes, usually, and always). Principal component analysis, using orthogonal and oblique rotations, was conducted to create a smaller set of components. Two items were removed because they loaded on multiple components. The remaining items loaded above 0.3 resulting in a 4-component solution (Table S3). An oblique rotation provided a better defined structure. The four components were: (1) Coping (three items related to regulation of negative emotions; e.g., feel it is easier to talk about feelings), (2) Mood Enhancer (three items related to positive emotions; e.g., feel happy), (3) Problem Behavior (three items related to behaviors resulting in negative effects; e.g., conflicts with police), and (4) Sex (two items related to sex life; e.g., feel more attractive). The components were statistically correlated ranging from 0.200 to 0.515 . Internal consistency for components was examined using Cronbach's alpha -0.839 for Coping, 0.822 for Mood Enhancer, 0.620 for Problem Behavior, and 0.774 for Sex. Although the index of reliability would be relatively low for short scales, they could possess acceptable psychometric quality [22]. Component scores were computed by taking the mean score of the items in each component.

\subsubsection{Reasons for Drinking}

Drinkers were asked to rate the importance of 11 reasons for drinking alcohol on a 4-point scale (not at all important, not very important, important, very important). Principal component analysis, using orthogonal and oblique rotations, was conducted to create a smaller set of components. The selection of items with loadings above 0.3 resulted in a 3-component solution (Table S4). An oblique rotation defined a more distinct structure. The components were: (1) Psychological Reasons (four items related to emotions; e.g., to help you relax), (2) Social Reasons (four items related to the social drinking environment; e.g., because others are drinking), and (3) Thirst/Taste (two items: because of thirst, and enjoy the taste). One item was omitted because of poor fit. The components were statistically correlated ranging from 0.192 to 0.400 . Internal consistency was examined using Cronbach's alpha -0.881 for Psychological Reasons, 0.694 for Social Reasons, and 0.591 for Thirst/Taste. Although the alpha for Thirst/Taste was lower than optimal, the scale has only two items. With two-item scales, common reliability estimators like Cronbach's Alpha may underestimate the true reliability of the scale (see discussion in [22]). Component scores were computed as the mean of the items in each component.

\subsection{Statistical Analysis}

For the comparisons between drinkers and abstainers (Part 1), means and standard deviation for the various scales were calculated. $t$-tests and adjusted $t$-tests (if the assumption of homogeneity of variance was not met) were used to test the group mean differences. Contingency tables were created to display the frequency distribution of the categorical variables by drinking status. Chi-square tests of association were used to test the relationship between categorical variables. Effect sizes for all of these tests were calculated-Cohen's $d$ for $t$-tests and Cramer's $V$ for chi-squares.

For the comparisons between the different drinking groups (Part 2), means and standard deviations were calculated. One-way analysis of variance (ANOVA) with post hoc multiple comparisons were used to test the significance of group mean differences. When the assumption of homogeneity of variance was not met, Welch's adjusted $F$ ratio was used. Contingency tables were created to display the frequency distribution of the categorical variables by four drinking 
groups. Chi-square tests of association were used to test the relationship between categorical variables. Effect sizes for all tests were calculated $-f$ for ANOVA and Cramer's $V$ for chi-square tests.

Data were analyzed using Statistical Package for the Social Sciences (SPSS) version 23 for Windows (SPSS Inc., Chicago, IL, USA).

\section{Results}

\subsection{Part 1: Comparisons between Drinkers and Abstainers}

\subsubsection{Demographic Variables of Drinkers Compared to Abstainers}

Of the 1640 interviews completed, 1467 (84.5\%) were valid and used in this study; $48 \%$ from drinkers and $52 \%$ from abstainers. The description of the sample is shown in Table 1. Chi-square tests were performed to test the relationship between drinking status and each demographic variable. Abstainers were more likely to be married, to have children, have a university education, or be associate-professionals or service workers. There were no drinking behavior differences by gender or age.

Table 1. Demographic comparison of Drinkers and Abstainers.

\begin{tabular}{|c|c|c|c|c|c|c|}
\hline & \multicolumn{2}{|c|}{ Drinkers } & \multicolumn{2}{|c|}{ Abstainers } & \multirow{2}{*}{$x^{2}$} & \multirow{2}{*}{ Cramer's $V$} \\
\hline & $n$ & $\%$ & $n$ & $\%$ & & \\
\hline Gender & & & & & 1.45 & 0.031 \\
\hline Male & 352 & 46.50 & 405 & 53.50 & & \\
\hline Female & 352 & 49.65 & 357 & 50.35 & & \\
\hline Age & & & & & 0.14 & 0.010 \\
\hline 18-24 years & 370 & 48.24 & 397 & 51.76 & & \\
\hline $25-29$ years & 178 & 47.21 & 199 & 52.79 & & \\
\hline 30-34 years & 156 & 48.45 & 166 & 51.55 & & \\
\hline Marital status & & & & & $9.49 *$ & 0.081 \\
\hline Married & 293 & 43.99 & 373 & 56.01 & & \\
\hline Divorced/separated/widowed & 6 & 75.00 & 2 & 25.00 & & \\
\hline Never married & 402 & 51.02 & 386 & 48.98 & & \\
\hline Have any children? & & & & & $9.68 *$ & 0.082 \\
\hline Yes & 233 & 43.15 & 307 & 56.85 & & \\
\hline No & 465 & 51.61 & 436 & 48.39 & & \\
\hline Education & & & & & $9.17 *$ & 0.079 \\
\hline University degree or higher & 255 & 44.58 & 317 & 55.42 & & \\
\hline Vocational/professional/non-university tertiary education & 389 & 51.73 & 363 & 48.27 & & \\
\hline Secondary school or less & 56 & 41.48 & 79 & 58.52 & & \\
\hline Currently a student? & & & & & $6.60 *$ & 0.067 \\
\hline Yes & 149 & 54.98 & 122 & 45.02 & & \\
\hline No & 551 & 46.34 & 638 & 53.66 & & \\
\hline Employment status & & & & & $9.36 *$ & 0.080 \\
\hline Employed & 490 & 45.97 & 576 & 54.03 & & \\
\hline Unemployed & 162 & 56.06 & 127 & 43.94 & & \\
\hline Other & 48 & 46.60 & 55 & 53.40 & & \\
\hline Occupation & & & & & $12.00 *$ & 0.095 \\
\hline Professionals & 204 & 50.50 & 200 & 49.50 & & \\
\hline Associate professionals & 98 & 43.95 & 125 & 56.05 & & \\
\hline Service workers & 190 & 44.71 & 235 & 55.29 & & \\
\hline Technicians & 55 & 52.38 & 50 & 47.62 & & \\
\hline Never employed & 109 & 58.29 & 78 & 41.71 & & \\
\hline
\end{tabular}

\subsubsection{Perceptions of Drinking of Drinkers Compared to Abstainers}

$T$-tests were used to test the group mean differences on each item. Drinkers and abstainers viewed drinking alcohol differently. Drinkers supported the relatively positive statements about alcohol—that 
drinking is one of life's pleasures and drinking with someone is a way of showing your friendship. Abstainers believed drinking had no benefits (Table 2).

\subsubsection{Acceptance of Drinking of Drinkers Compared to Abstainers}

$T$-tests were also used to test the component mean differences between drinkers and abstainers. Significant $t$ values indicate that abstainers, on average, rated the acceptance of drinking to be lower than drinkers for each drinking situation (Table 2).

Table 2. Perception of drinking and acceptance of drinking by drinking status.

\begin{tabular}{|c|c|c|c|c|c|c|}
\hline & \multicolumn{2}{|c|}{ Drinkers } & \multicolumn{2}{|c|}{ Abstainers } & \multirow{2}{*}{$t$} & \multirow{2}{*}{ Cohen's $d$} \\
\hline & Mean & SD & Mean & SD & & \\
\hline \multicolumn{7}{|l|}{ Perception of drinking } \\
\hline \multicolumn{7}{|l|}{ Item } \\
\hline One of life's pleasures & 3.37 & 1.04 & 2.92 & 0.99 & $-8.50 * *$ & 0.448 \\
\hline Drinking with someone is a way of showing friendship & 3.89 & 0.78 & 3.28 & 0.98 & $-13.25^{* *}$ & 0.706 \\
\hline Drinking has no benefits & 3.28 & 1.01 & 3.65 & 0.92 & $7.43 * *$ & 0.396 \\
\hline \multicolumn{7}{|l|}{ Acceptance of drinking } \\
\hline \multicolumn{7}{|l|}{ Component } \\
\hline With Young Children & 1.55 & 0.64 & 1.35 & 0.61 & $-5.77 * *$ & 0.313 \\
\hline Adult Private Context & 2.29 & 0.76 & 2.07 & 0.70 & $-5.54 * *$ & 0.304 \\
\hline Social Drinking for Males & 2.38 & 0.62 & 2.11 & 0.63 & $-8.20 * *$ & 0.437 \\
\hline Social Drinking for Females & 2.08 & 0.61 & 1.86 & 0.68 & $-6.40^{* *}$ & 0.343 \\
\hline
\end{tabular}

Notes: Perception of drinking scale: 1 = strongly disagree, 5 = strongly agree; Acceptance of drinking scale: 1 = should not drink; 2 = can drink a little but without any real effects, 3 = can drink and feel some effects but not get intoxicated, $4=$ getting intoxicated sometimes is $\mathrm{OK}, 5=$ getting intoxicated is always $\mathrm{OK}$. ${ }^{* *} p<0.001$.

\subsubsection{Reasons for Not Drinking of Drinkers Compared to Abstainers}

A chi-square test of association shows that respondents who thought the given reasons were important were more likely to be abstainers (Table 3).

Table 3. Reasons for not drinking by drinking status.

\begin{tabular}{|c|c|c|c|c|c|c|}
\hline \multirow{3}{*}{ Cluster } & \multicolumn{2}{|c|}{ Drinkers } & \multicolumn{2}{|c|}{ Abstainers } & \multirow{2}{*}{$x^{2}$} & \multirow{2}{*}{ Cramer's $V$} \\
\hline & \multirow{2}{*}{$n$} & \multirow{2}{*}{$\%$} & \multirow{2}{*}{$n$} & \multirow{2}{*}{$\%$} & & \\
\hline & & & & & $73.54 * *$ & 0.257 \\
\hline Most reasons for not drinking are: & & & & & & \\
\hline Not very important-unimportant (low-rating group) & 211 & 60.98 & 135 & 39.02 & & \\
\hline Importance varied by reason (mid-range group) & 260 & 57.78 & 190 & 42.22 & & \\
\hline Important-very important (high-rating group) & 97 & 30.79 & 218 & 69.21 & & \\
\hline
\end{tabular}

\subsection{Part 2: Comparisons among Four Drinking Frequency-Quantity Groups}

\subsubsection{Usual Drinking Place of Drinkers in the Sample}

Drinking behavior is usually shaped by the drinking environment. In the past 12 months, more than $70 \%$ of the drinkers reported drinking at restaurants for dinner, at a friends' homes with friends, and at home with friends. Thirty percent drank at bars, and only $14 \%$ of drinkers reported drinking with friends in public places like a park.

\subsubsection{Demographic Variables Associated with Drinking Frequency and Quantity}

Among drinkers, 28.7\% were in the LoF-LoQ group, 23.7\% were in the HiF-LoQ group, $19.6 \%$ were in the LoF-HiQ group, and the remaining $28 \%$ were in the HiF-HiQ group. The demographic description of those drinking groups is presented in Table 4 . Chi-square tests were used to test the 
demographic differences among drinker groups. Drinking behavior was related to gender, whether or not they had children, their highest education level, employment status, and occupation. Male drinkers were more likely to be in the LoF-LoQ or HiF-LoQ groups, whereas female drinkers were more likely to be in the LoF-HiQ or HiF-HiQ groups. Drinkers who had children were more likely to be HiF-HiQ drinkers, whereas drinkers who were not parents were more likely to be LoF-LoQ drinkers. Drinkers who received secondary school education or less were more likely to be in the HiF-LoQ group. In contrast, higher educated drinkers were more likely to be LoF-LoQ drinkers. Unemployed drinkers were more likely to be in the LoF-LoQ group than employed drinkers. Professionals and service workers were more likely to be HiF-HiQ drinkers, associate professionals were likely to be HiF-LoQ drinkers, and technicians and never employed people were likely to be LoF-LoQ drinkers.

\subsubsection{Perception of Drinking Associated with Drinking Frequency and Quantity}

One-way ANOVAs with post-hoc multiple comparisons were used to test the significance of group mean differences. We found that the perceptions of drinking differed by group drinking frequency (Table 5). HiF-LoQ drinkers were more likely than LoF-LoQ drinkers to see drinking as one of life's pleasures and to see drinking with someone as a way to show friendship. Similarly, HiF-HiQ drinkers were more likely than LoF-HiQ drinkers to agree that drinking is one of life's pleasures and a way of showing friendship. There was no difference between drinking groups on the statement that drinking had no benefits.

\subsubsection{Acceptance of Drinking Associated with Drinking Frequency and Quantity}

Table 5 shows results from one-way ANOVAs with post-hoc multiple comparisons performed to examine whether the component mean scores on the acceptance of drinking in various situations differed between drinking groups. Acceptance of drinking varied within drinkers in different drinking situations. When spending time with young children, HiF-LoQ drinkers were more likely to approve of drinking than LoF-LoQ and LoF-HiQ drinkers, but HiF-HiQ drinkers had a similar degree of approval as LoF-LoQ and LoF-HiQ drinkers. During social drinking, HiF-HiQ drinkers reported higher acceptance for both male and female drinking. All drinkers felt that higher alcohol consumption was more acceptable for men than for women.

\subsubsection{Effects of Drinking Associated with Drinking Frequency and Quantity}

Table 6 shows results of one-way ANOVAs with post-hoc multiple comparisons of the effects of drinking by drinking groups. LoF-LoQ drinkers consistently had lower mean scores on the effects of drinking scale than HiF-HiQ drinkers. In addition, positive effects from drinking were more frequently identified than negative effects by all drinkers.

\subsubsection{Reasons for Drinking Associated with Drinking Frequency and Quantity}

Table 6 shows the results of one-way ANOVAs with post-hoc multiple comparisons used to test group differences in reasons for drinking. The LoF-LoQ drinkers consistently gave lower scores for importance on all of the reasons for drinking compared to the HiF-HiQ drinkers. Members of all four drinking groups rated Social Reasons for drinking more important than Psychological Reasons and more important than Thirst/Taste.

\subsubsection{Reasons for Not Drinking Associated with Drinking Frequency and Quantity}

A non-significant chi-square indicates that drinkers' rating of the reasons for not drinking did not differ across drinking groups (Table 7). 
Table 4. Demographic descriptions by drinking frequency-quantity groups.

\begin{tabular}{|c|c|c|c|c|c|c|c|c|c|c|}
\hline & \multicolumn{2}{|c|}{$\begin{array}{l}\text { Low Frequency-Low } \\
\text { Quantity (LoF-LoQ) }\end{array}$} & \multicolumn{2}{|c|}{$\begin{array}{l}\text { High Frequency-Low } \\
\text { Quantity (HiF-LoQ) }\end{array}$} & \multicolumn{2}{|c|}{$\begin{array}{l}\text { Low Frequency-High } \\
\text { Quantity (LoF-HiQ) }\end{array}$} & \multicolumn{2}{|c|}{$\begin{array}{l}\text { High Frequency-High } \\
\text { Quantity (HiF-HiQ) }\end{array}$} & \multirow{2}{*}{$x^{2}$} & \multirow{2}{*}{ Cramer's $V$} \\
\hline & $n$ & $\%$ & $n$ & $\%$ & $n$ & $\%$ & $n$ & $\%$ & & \\
\hline Gender & & & & & & & & & $58.58^{* *}$ & 0.289 \\
\hline Male & 117 & 33.24 & 113 & 32.10 & 37 & 10.51 & 85 & 24.15 & & \\
\hline Female & 85 & 24.22 & 54 & 15.39 & 100 & 28.49 & 112 & 31.91 & & \\
\hline Age & & & & & & & & & 4.09 & 0.054 \\
\hline $18-24$ years & 117 & 31.54 & 87 & 23.45 & 71 & 19.14 & 96 & 25.88 & & \\
\hline 25-29 years & 44 & 25.00 & 42 & 23.86 & 34 & 19.32 & 56 & 31.82 & & \\
\hline 30-34 years & 40 & 25.81 & 38 & 24.52 & 32 & 20.65 & 45 & 29.03 & & \\
\hline Marital status & & & & & & & & & 5.27 & 0.087 \\
\hline Married & 71 & 24.40 & 70 & 24.05 & 60 & 20.62 & 90 & 30.93 & & \\
\hline Never married & 128 & 32.00 & 93 & 23.25 & 76 & 19.00 & 103 & 25.75 & & \\
\hline Have any children? & & & & & & & & & $10.31 *$ & 0.122 \\
\hline Yes & 49 & 21.21 & 56 & 24.24 & 52 & 22.51 & 74 & 32.03 & & \\
\hline No & 150 & 32.26 & 111 & 23.87 & 85 & 18.28 & 119 & 25.59 & & \\
\hline Education & & & & & & & & & $13.31 *$ & 0.098 \\
\hline University degree or higher & 75 & 29.64 & 58 & 22.92 & 51 & 20.16 & 69 & 27.27 & & \\
\hline Vocational/professional/non-university tertiary education & 118 & 30.49 & 86 & 22.22 & 75 & 19.38 & 108 & 27.91 & & \\
\hline Secondary school or less & 7 & 12.50 & 22 & 39.29 & 8 & 14.29 & 19 & 33.93 & & \\
\hline Currently a student? & & & & & & & & & 4.72 & 0.082 \\
\hline Yes & 49 & 32.89 & 31 & 20.81 & 34 & 22.82 & 35 & 23.49 & & \\
\hline No & 148 & 26.96 & 136 & 24.77 & 103 & 18.76 & 162 & 29.51 & & \\
\hline Employment status & & & & & & & & & $15.48^{*}$ & 0.106 \\
\hline Employed & 128 & 26.28 & 132 & 27.10 & 90 & 18.48 & 137 & 28.13 & & \\
\hline Unemployed & 59 & 36.42 & 27 & 16.67 & 34 & 20.99 & 42 & 25.93 & & \\
\hline Other & 10 & 21.74 & 7 & 15.22 & 13 & 28.26 & 16 & 34.78 & & \\
\hline Occupation & & & & & & & & & $35.44^{* *}$ & 0.135 \\
\hline Professionals & 56 & 27.59 & 39 & 19.21 & 32 & 15.76 & 76 & 37.44 & & \\
\hline Associate professionals & 28 & 28.87 & 30 & 30.93 & 19 & 19.59 & 20 & 20.62 & & \\
\hline Service workers & 38 & 20.00 & 49 & 25.79 & 46 & 24.21 & 57 & 30.00 & & \\
\hline Technicians & 18 & 33.33 & 14 & 25.93 & 7 & 12.96 & 15 & 27.78 & & \\
\hline Never employed & 42 & 39.62 & 22 & 20.75 & 26 & 24.53 & 16 & 15.09 & & \\
\hline
\end{tabular}

Notes: LoF-LoQ $=$ drinks less than one time a month and not more than 1 drink for women $/ 2$ drinks for men at one time, HiF-LoQ $=$ drinks more than one time a month but not more than 1 drink for women $/ 2$ drinks for men at one time, $\mathrm{LoF}-\mathrm{HiQ}=$ drinks less than one time a month but drinks more than 1 drink for women $/ 2$ drinks for men at one time, and HiF-HiQ = drinks more than one time a month and drinks more than 1 drink for women $/ 2$ drinks for men at one time. ${ }^{* *} p<0.001 ;{ }^{*} p<0.05$. 
Table 5. Perception of drinking and acceptance of drinking by drinking groups.

\begin{tabular}{|c|c|c|c|c|c|c|c|c|c|c|}
\hline & \multicolumn{2}{|c|}{$\begin{array}{l}\text { Low Frequency-Low } \\
\text { Quantity (LoF-LoQ) }\end{array}$} & \multicolumn{2}{|c|}{$\begin{array}{l}\text { High Frequency-Low } \\
\text { Quantity (HiF-LoQ) }\end{array}$} & \multicolumn{2}{|c|}{$\begin{array}{l}\text { Low Frequency-High } \\
\text { Quantity (LoF-HiQ) }\end{array}$} & \multicolumn{2}{|c|}{$\begin{array}{l}\text { High Frequency-High } \\
\text { Quantity (HiF-HiQ) }\end{array}$} & \multirow[t]{2}{*}{$F$} & \multirow[t]{2}{*}{$f$} \\
\hline & Mean & SD & Mean & SD & Mean & SD & Mean & SD & & \\
\hline \multicolumn{11}{|l|}{ Perceptions of drinking } \\
\hline Item & & & & & & & & & & \\
\hline one of life's pleasures & $3.21_{\mathrm{a}, \mathrm{b}}$ & 1.12 & $3.56_{a, c}$ & 0.98 & $3.00_{\mathrm{c}, \mathrm{d}}$ & 1.01 & $3.66_{\mathrm{b}, \mathrm{d}}$ & 0.89 & $15.88^{* *}$ & 0.264 \\
\hline drinking with someone is a way of showing friendship & $3.74_{e, f}$ & 0.87 & $3.95 \mathrm{e}$ & 0.78 & $3.77_{\mathrm{g}}$ & 0.77 & $4.10_{\mathrm{f}, \mathrm{g}}$ & 0.64 & $10.35 * *$ & 0.195 \\
\hline drinking has no benefits & 3.25 & 0.98 & 3.26 & 1.05 & 3.47 & 1.05 & 3.18 & 0.96 & 2.38 & 0.110 \\
\hline \multicolumn{11}{|l|}{ Acceptance of drinking } \\
\hline \multicolumn{11}{|l|}{ Component } \\
\hline With Young Children & $1.46_{\mathrm{h}}$ & 0.61 & $1.73_{\mathrm{h}, \mathrm{i}}$ & 0.69 & $1.43_{\mathrm{i}}$ & 0.64 & 1.58 & 0.60 & $7.59 * *$ & 0.190 \\
\hline Adult Private Context & 2.22 & 0.75 & 2.30 & 0.71 & 2.23 & 0.71 & 2.41 & 0.81 & 2.34 & 0.105 \\
\hline Social Drinking for Males & $2.20_{\mathrm{j}, \mathrm{k}}$ & 0.55 & $2.40_{\mathrm{j}, 1}$ & 0.60 & $2.28_{\mathrm{m}}$ & 0.56 & $2.62_{\mathrm{k}, 1, \mathrm{~m}}$ & 0.65 & $17.25^{* *}$ & 0.268 \\
\hline Social Drinking for Females & $1.95_{\mathrm{n}}$ & 0.55 & $2.09_{\mathrm{o}}^{\prime \prime 2}$ & 0.58 & $2.02_{\mathrm{p}}$ & 0.62 & $2.28_{\mathrm{n}, \mathrm{o}, \mathrm{p}}$ & 0.64 & $9.93^{* *}$ & 0.208 \\
\hline
\end{tabular}

Notes: LoF-LoQ = drinks less than one time a month and not more than 1 drink for women $/ 2$ drinks for men at one time, HiF-LoQ = drinks more than one time a month but not more than 1 drink for women $/ 2$ drinks for men at one time, LoF-HiQ = drinks less than one time a month but drinks more than 1 drink for women $/ 2$ drinks for men at one time, and HiF-HiQ = drinks more than one time a month and drinks more than 1 drink for women $/ 2$ drinks for men at one time. Means sharing a common subscript are statistically different at $\alpha=0.05$ according to the Tukey HSD procedure. Perception of drinking scale: $1=$ strongly disagree, $5=$ strongly agree; Acceptance of drinking scale: $1=$ should not drink; $2=$ can drink a little but without any real effects, 3 = can drink and feel some effects but not get intoxicated, $4=$ getting intoxicated sometimes is OK, $5=$ getting intoxicated is always OK. ${ }^{* *} p<0.001$. 
Table 6. Effects of drinking and Reasons for drinking by drinking level groups.

\begin{tabular}{|c|c|c|c|c|c|c|c|c|c|c|}
\hline & \multicolumn{2}{|c|}{$\begin{array}{l}\text { Low Frequency-Low } \\
\text { Quantity (LoF-LoQ) }\end{array}$} & \multicolumn{2}{|c|}{$\begin{array}{l}\text { High Frequency-Low } \\
\text { Quantity (HiF-LoQ) }\end{array}$} & \multicolumn{2}{|c|}{$\begin{array}{l}\text { Low Frequency-High } \\
\text { Quantity (LoF-HiQ) }\end{array}$} & \multicolumn{2}{|c|}{$\begin{array}{l}\text { High Frequency-High } \\
\text { Quantity (HiF-HiQ) }\end{array}$} & \multirow[t]{2}{*}{$F$} & \multirow[t]{2}{*}{$f$} \\
\hline & Mean & SD & Mean & SD & Mean & SD & Mean & SD & & \\
\hline \multicolumn{11}{|l|}{ Effects of drinking } \\
\hline \multicolumn{11}{|l|}{ Component } \\
\hline Coping & $2.29 \mathrm{a}, \mathrm{b}$ & 0.89 & $2.72_{a}$ & 0.96 & $2.50_{c}$ & 0.98 & $2.85_{\mathrm{b}, \mathrm{c}}$ & 1.00 & $12.17^{* *}$ & 0.223 \\
\hline Mood Enhancer & $2.76_{\mathrm{d}, \mathrm{e}}$ & 0.87 & $3.03_{\mathrm{d}}$ & 0.80 & $2.86_{\mathrm{f}}$ & 0.88 & $3.26_{\mathrm{e}, \mathrm{f}}$ & 0.86 & $12.04 * *$ & 0.225 \\
\hline Problem Behavior & $1.29_{\mathrm{g}}$ & 0.48 & 1.37 & 0.52 & 1.33 & 0.52 & $1.44_{\mathrm{g}}$ & 0.50 & $2.79 *$ & 0.109 \\
\hline Sex & $1.61_{\mathrm{h}, \mathrm{i}}^{8}$ & 0.75 & $2.23_{h, j}$ & 0.93 & $1.55_{\mathrm{j}, \mathrm{k}}$ & 0.88 & $2.07_{\mathrm{i}, \mathrm{k}}^{\mathrm{b}}$ & 1.03 & $15.06^{* *}$ & 0.321 \\
\hline \multicolumn{11}{|l|}{ Reasons for drinking } \\
\hline Component & & & & & & & & & & \\
\hline Psychological Reasons & $1.95_{1, \mathrm{~m}}$ & 0.71 & $2.20_{1}$ & 0.65 & 2.09 & 0.74 & $2.26_{\mathrm{m}}$ & 0.71 & $7.01 * *$ & 0.169 \\
\hline Social Reasons & $2.59_{n, o, p}$ & 0.58 & $2.76_{n}$ & 0.47 & $2.78_{\mathrm{o}}$ & 0.55 & $2.88_{p}$ & 0.56 & $8.85^{* *}$ & 0.186 \\
\hline Thirst/Taste & $1.48_{\mathrm{q}}$ & 0.50 & 1.59 & 0.61 & 1.54 & 0.53 & $1.64_{\mathrm{q}}$ & 0.63 & $2.85^{*}$ & 0.105 \\
\hline
\end{tabular}

Notes: LoF-LoQ = drinks less than one time a month and not more than 1 drink for women/2 drinks for men at one time, HiF-LoQ = drinks more than one time a month but not more than 1 drink for women $/ 2$ drinks for men at one time, LoF-HiQ = drinks less than one time a month but drinks more than 1 drink for women $/ 2$ drinks for men at one time, and HiF-HiQ $=$ drinks more than one time a month and drinks more than 1 drink for women $/ 2$ drinks for men at one time. Means sharing a common subscript are statistically different at $\alpha=0.05$ according to the Tukey HSD procedure. Effects of drinking scale: 1 = seldom, $5=$ always; Reasons for drinking scale: $1=$ not at all important, $4=$ very important. ${ }^{* *} p<0.001 ;{ }^{*} p<0.05$.

Table 7. Reasons for not drinking by drinking groups.

\begin{tabular}{|c|c|c|c|c|c|c|c|c|c|c|}
\hline & \multicolumn{2}{|c|}{$\begin{array}{l}\text { Low Frequency-Low } \\
\text { Quantity (LoF-LoQ) }\end{array}$} & \multicolumn{2}{|c|}{$\begin{array}{l}\text { High Frequency-Low } \\
\text { Quantity (HiF-LoQ) }\end{array}$} & \multicolumn{2}{|c|}{$\begin{array}{l}\text { Low Frequency-High } \\
\text { Quantity (LoF-HiQ) }\end{array}$} & \multicolumn{2}{|c|}{$\begin{array}{l}\text { High Frequency-High } \\
\text { Quantity (HiF-HiQ) }\end{array}$} & \multirow{2}{*}{$x^{2}$} & \multirow{2}{*}{ Cramer's V } \\
\hline & $n$ & $\%$ & $n$ & $\%$ & $n$ & $\%$ & $n$ & $\%$ & & \\
\hline $\begin{array}{l}\text { Cluster } \\
\text { Most reasons for not drinking are: }\end{array}$ & & & & & & & & & 5.26 & 0.068 \\
\hline not very important-unimportant (low-rating group) & 63 & 29.86 & 45 & 21.33 & 44 & 20.85 & 59 & 27.96 & & \\
\hline importance varied by reason (mid-range group) & 66 & 25.48 & 67 & 25.87 & 52 & 20.08 & 74 & 28.57 & & \\
\hline important-very important (high-rating group) & 32 & 32.99 & 26 & 26.80 & 20 & 20.62 & 19 & 19.59 & & \\
\hline
\end{tabular}

Notes: LoF-LoQ = drinks less than one time a month and not more than 1 drink for women $/ 2$ drinks for men at one time, HiF-LoQ = drinks more than one time a month but not more than 1 drink for women $/ 2$ drinks for men at one time, LoF-HiQ = drinks less than one time a month but drinks more than 1 drink for women $/ 2$ drinks for men at one time, and HiF-HiQ = drinks more than one time a month and drinks more than 1 drink for women $/ 2$ drinks for men at one time. 


\section{Discussion}

This paper is based on data from a community sample of young people aged 18-34 in the city of Wuhan, China. With probability sampling of a wider age range of young people, this paper compliments previous studies of young people in China, which were largely limited to samples of higher education students $[13,14]$. Since there is no prior data on drinking in this age group, there really is no way to know in advance what the possible confounders would be. Our goal was to shed some light on drinking patterns and beliefs that would need to be considered in future research. Some of the findings from this study are not consistent with earlier studies reported in the published literature. For a comparison of this sample of 18-34 year olds in Wuhan, China to with the same-age group from three other countries, see Taylor et al. [15].

This analysis of data from Chinese young adults found that gender and age (18-34 years) were not associated with whether someone drank or not. Literature on alcohol use in China has consistently reported higher drinking for males compared to females (e.g., [1,6,7,9]). Another common observation has been drinking rates increasing with age, reaching a peak in middle age $[2,10]$. Observations by our research team in China suggest that it is becoming increasingly acceptable for females to admit to drinking, perhaps leading to similar rates of drinking for men and women in this survey compared to earlier studies. Women are also increasingly a part of the employed workforce, which exposes them to workplace drinking practices.

While there was no gender difference in drinking versus abstaining, gender differences were identified in the Frequency-Quantity group analysis. Men were more likely than women to be in the low-quantity groups (LoF-LoQ and HiF-LoQ). This finding contradicted our observations of drinking culture in China, where men are often forced to drink competitively and women are not. It is possible that this survey has detected changing drinking patterns related to gender and suggests that previous generalizations based on only gender and drinking/nondrinking categories may conceal differences in quantity and frequency of drinking that are important for a better understanding of drinking behavior. We based our classifications of drinking quantity on the official Dietary Guidelines for Chinese Residents, which defined "moderate drinking" as no more than two drinks at one time for males and no more than one drink at one time for females. The different cut-off points explains why the majority of the 18- to-34-year-old females in this survey were in the high-quantity groups (LoF-HiQ and HiF-HiQ) and the majority of the 18- to 34-year old males were in the low-quantity groups (LoF-LoQ and HiF-LoQ). A different result would have come from using a different definition for moderate drinking. We acknowledge that the drinking definitions used are conservative by Western standards, but they are consistent with Chinese drinking culture. Taylor et al.'s four-country study [15] found a comparatively low rate of excessive drinking for the China sample compared to the other three country samples. It is also important to keep in mind that $40 \%$ of the China sample reported drinking spirits, which in China range in strength from $40 \%$ to $60 \%$ alcohol by volume.

The 18- to 34-year-olds with vocational/professional (non-university) education, as a group, had the fewest abstainers compared to those who had university degrees or those who had secondary or less education. $\mathrm{Wu}$ and colleagues [4] report that in China higher education levels were related to lower drinking rates, while others indicate the opposite [2,3]. Previous studies and our results do not present a clear picture of the relationship between education and drinking.

We found that professionals and technicians were more likely to be drinkers than associate professionals and service workers. This is consistent with results reported by Li and colleagues [3] who suggested higher drinking rates for workers in the more technical manufacturing and construction industry than those in the less technical service sector. Assuming more education is required for the more technical occupations, this finding appears to contradict earlier studies that found higher levels of education associated with lower levels of drinking. Wu and colleagues [4] found no significant difference in the proportion of regular alcohol drinkers across different occupations among rural or urban respondents. Unfortunately different occupation categories were used in the different studies, and not all survey-designed occupational categories correlate to education levels. Thus, a unified set 
of occupation categories is needed to truly assess the relationship between occupation, education, and drinking behavior.

The present study indicated that married respondents and respondents who had a child were more likely to be abstainers. This finding contrasts with findings reported in earlier Chinese literature which reported that being married was associated with higher drinking rates [2-4]. Married couples planning to start a family may be benefitting from the increase in community health information about the risks of alcohol on pregnancy, especially among the higher educated Chinese. Married people with children may have benefited from clinic-based education services associated with childbirth and well-baby clinics. Interestingly, even though people with children were more likely to be abstainers, the drinkers with children were more likely to fall into the HiF-HiQ category, especially those employed as professionals, service workers, and technicians. It suggests that young people with children are also employed in occupations that require business drinking and toasting for job protection and promotion [23]. At workplace banquets and celebrations, subordinates are expected to toast their superiors, often more than once. Emptying the entire glass of alcohol indicates the highest respect during a toast. The result of toasting etiquette is both heavy and frequent drinking.

In addition to the demographic differences among the four drinking frequency-quantity groups, drinking experience seemed to influence drinking attitudes and motives. The present study consistently found that drinkers were more likely to agree that drinking is one of life's pleasures and that drinking with someone is a way of showing friendship. Drinkers were more likely than abstainers to approve alcohol use in all four situations studied-where young children are present, in adult private context, in social drinking for men, and in social drinking for women. Frequent drinkers (HiF-LoQ and HiF-HiQ), in particular, had even stronger positive feelings about alcohol use than non-frequent drinkers (LoF-LoQ and LoF-HiQ). Positive attitudes were associated with both increased frequency of drinking and higher acceptance of others' drinking. Other researchers have drawn this same conclusion. Wang and colleagues [1] reported that medical students with more positive attitudes toward alcohol drinking were more likely to consume alcohol. Luo and colleagues [7] noted that drinkers were more likely to approve others' drinking behavior and drunkenness behavior than abstainers. Among college students in Qinghai, drinkers were less likely to have negative feeling about women drinking [6]. Even though drinkers were more accepting of other people's drinking than abstainers, both drinkers and abstainers thought that drinking was not acceptable when parents were with their young children. Both groups rated social drinking among females less acceptable than social drinking among males and drinking in an adult private context, such as with a spouse, partner, or cohabitant.

The present study indicated that social motives were more important than psychological motives for drinking. Studies in China consistently reported that needs of social communication, celebrations, and coping were commonly-given reasons for drinking among college students $[1,8,9]$. The importance of drinking together to reinforce friendships and social networks is deeply grounded in traditional Chinese culture.

In this study, the high-frequency drinkers (HiF-LoQ and HiF-HiQ) were more likely to report coping with negative emotions was an effect of their drinking alcohol compared to low-frequency drinkers (LoF-LoQ and LoF-HiQ). The combination of social obligations to drink and expecting positive outcomes like mood enhancement reinforces drinkers and may lead them to drink more frequently and in higher quantities.

Drinkers who participated in this survey reported the majority of their drinking was at restaurants for dinner, at a friends' homes with friends, and at home with friends-environments that include other people. Social reasons appeared to be the main reason for drinking for all four drinking behavior groups. These findings confirm that in China drinking plays an important role in socializing, and that celebrations and festivities are typically accompanied with alcohol. Much drinking behavior research in the West is based on models and theories of behavior change that assume individualized decision-making about drinking based on experiences and outcome expectations. Clearly this is not 
the only way to view and understand alcohol use. Meier, Warde and Holmes [24] recognize the limitations of these individualized models and theories, and they suggest social practice theory as a complementary approach that studies drinking in the context of activities that preceded the drinking event and those that followed and the encompassing physical and social circumstances. Rather than seeing alcohol is a unitary behavior they suggest it be viewed as a practice occurring within a much larger context that incorporated cultural tradition, local values, the social and the physical environment and the expectations of what follows the drinking event. We totally agree and believe the findings from this study, especially those that do not concur with previous findings, suggest the need for alternative ways of viewing alcohol drinking.

\section{Limitations}

This study is limited to one city: Wuhan. How similar to other cities of the same size is unknown. The data are cross-sectional in nature, thus causal inferences are not suggested. The fact that $90 \%$ of the sample reported education beyond secondary school suggests this may be an atypical city. While this study explored a variety of dimensions of drinking it asked few questions about important drinking contexts such as the workplace. This analysis is based on self-reported alcohol use/nonuse, which could be unreliable. To improve reliability the data were gathered in face-to-face interviews to reduce misunderstandings. Also, in China, there is less cultural stigma and fewer legal prohibitions on alcohol use, compared to western countries, so there was less reason for participants to underreport alcohol use. The questions on the scales used were unique to this study so comparisons are not possible. While the sample size was large, it was not large enough to explore in detail the role of the possible covariates or additional factors because the cell sizes became too small, leading to a loss of statistical power and making interpretations tenuous. Based on these results and results from other studies, future studies could be built around hypotheses of causal relationships allowing the exploration of confounding variables. Nevertheless this study did provide useful information about alcohol drinking and abstaining in the population of 18- to 34-year olds (not just students) and did use measures worthy of further refinement for future studies.

Former drinkers were excluded in this study due to small sample size and lack of information about drinking history. Former drinkers are a special group who had experience in drinking but currently do not drink. They may have a more similar attitude toward drinking to current drinkers than to lifetime abstainers. It would be worthwhile to examine the similarities and differences between former drinkers and current drinkers and lifetime abstainers in future studies.

\section{Conclusions}

These findings of this descriptive study confirm that in China drinking plays an important role in socializing, and that celebrations and festivities are typically accompanied with alcohol. Western models of individualized motivation of behaviors may not accurately explain alcohol use in China that emphasize collective values and relationships. The questions and methods used generated useful data and should be reviewed and possibly revised for use with other samples from communities from across China. Larger samples would accommodate more sophisticated analyses. This would generate a more representative picture of drinking patterns in this age group. The findings from the study, especially those that do not concur with previous findings, suggest the need for further study.

Supplementary Materials: The following are available online at http:/ / www.mdpi.com/1660-4601/15/8/1675/s1, Table S1: Loadings for four-oblique components for "acceptance of drinking" $(N=1499)$, Table S2: Reasons for not drinking cluster description $(N=1221)$, Table S3: Loadings on three-oblique components for "effects of drinking" $(N=548)$, Table S4: Loadings on three-oblique components for "reasons for drinking" $(N=790)$.

Author Contributions: Secondary data analysis design and methodology, I.M.N., L.-w.Y., D.F.S.; formal analysis, L.-w.Y.; Writing-original draft preparation, L.-w.Y.; Writing-review and editing, L.Q., I.M.N., L.-w.Y., D.F.S., J.X.; Original project administration, J.X.

Funding: The preparation of this manuscript received no external funding. 
Acknowledgments: De-identified data were used by permission from Taylor et al. [15]. A.W Taylor and B.M Bewick led the work that resulted in the instrument used in this study. Data collection was funded by the International Alliance for Responsible Drinking. The funders had no role in the analysis, data interpretation or writing of this manuscript.

Conflicts of Interest: Prior to 2015, L.Q. was a member of an advisory committee of the International Center on Alcohol Policy, Washington, D.C. (now known as the International Alliance for Responsible Drinking), and received fees and travel support to attend and to speak at meetings sponsored or cosponsored by ICAP. Through a grant from ICAP to The Buffalo Beach Company, Lincoln, NE, USA, L.Q. received fees for assisting in the organization of and data collection for projects funded by ICAP. Her regular salary was paid by the Chinese government through the Chinese Center for Health Education. Funding to support L.Q.'s work for the collection of the China data analyzed in this manuscript including travel and reasonable expenses was provided by International Alliance for Responsible Drinking. Prior to 2014, I.M.N. was a member of the Research Advisory Committee of the International Center for Alcohol Policies, Washington, D.C. Prior to 2014 he received fees and travel support to attend meetings sponsored or co-sponsored by the International Center for Alcohol Policies. Prior to 2013, I.M.N. consulted for the National Health Education Institute, China CDC, Chinese Center for Health Education (CCHE)/Health News \& Communication Center, Ministry of Health (HNCC, MOH). In the past 5 years, he has received University of Nebraska employment-related funding from the U.S. Department of Education, the Nebraska Department of Health and Human Services, the Nebraska Department of Transportation/Office of Highway Safety. As American deputy director of the American Exchange Center at Xi'an Jiaotong University from 2012-2015, I.M.N. received funds from the US State Department and the University of Nebraska. I.M.N. owns an equity interest in The Buffalo Beach Company (Lincoln, NE, USA) and has received consulting fees from The Buffalo Beach Company for independent research related to indigenous alcohol use, traffic safety, and evaluation of alcohol risk reduction programs. L.-W.Y., D.F.S., \& J.X. have no potential competing interests to declare.

\section{References}

1. Wang, P.; Zhao, M.; Li, H.; Yuan, L.; Yang, J. Investigations on the alcohol using behaviors and the influencing factors among medical college students. Chin. J. School Health 2005, 26, 360-361. (In Chinese)

2. Li, Y.; Jiang, Y.; Zhang, M.; Yin, P.; Wu, F.; Zhao, W. Drinking behavior among men and women in China: The 2007 China Chronic Disease and Risk Factor Surveillance. Addiction 2011, 106, 1946-1956. [CrossRef] [PubMed]

3. Li, Y.; Zhang, M.; Jiang, Y.; Deng, Q.; Zhao, Y.; Huang, Z.; Zeng, X.; Wang, L. Drinking behaviors and patterns among floating population aged 18-59 years old in China. Chin. J. Epidemiol. 2014, 35, 1186-1191. (In Chinese)

4. Wu, B.; Mao, Z.-F.; Rockett, I.R.H.; Yue, Y. Socioeconomic status and alcohol use among urban and rural residents in China. Subst. Use Misuse 2008, 43, 952-966. [CrossRef] [PubMed]

5. Zhang, J.; Wang, J.; Lu, Y.; Qiu, X.; Fang, Y. Alcohol abuse in a metropolitan city in China: A study of the prevalence and risk factors. Addiction 2004, 99, 1103-1110.

6. Du, W.; Zhao, X.; Li, B.; Zhang, H.; Liu, S.; Mao, H. Analysis of alcohol drinking and its influence factors of college students in Qinghai Province. Chin. J. Dis. Control. Prev. 2015, 9, 1070-1071, 1074. (In Chinese)

7. Luo, D.; Tang, H.; Chen, W.; Pang, X. Investigation on the alcohol drinking behaviors among medical college students. Modern Prev. Med. 2011, 38, 292-293, 297. (In Chinese)

8. Wu, S.; Xu, R.; Zhang, J.; Wang, Z.; Li, K.; Yan, S.; Lin, X. Status of smoking and drinking among college students in Beijing. Chin. J. School Health 2009, 30, 18-19. (In Chinese)

9. Zhang, X.; Liu, F. Analysis of the drinking status among college students and the influencing factors. Modern Prev. Med. 2008, 35, 534-536. (In Chinese)

10. Hao, W.; Su, Z.; Liu, B.; Zhang, K.; Yang, H.; Chen, S.; Biao, M.; Cui, C. Drinking and drinking patterns and health status in the general population of five areas of China. Alcohol Alcohol. 2004, 39, 43-52. [CrossRef] [PubMed]

11. Cheng, H.G.; Deng, F.; Xiong, W.; Phillips, M.R. Prevalence of alcohol use disorders in mainland China: A systematic review. Addiction 2015, 110, 761-774. [CrossRef] [PubMed]

12. Phillips, M.R.; Cheng, H.G.; Li, X.; Zhang, J.; Shi, Q.; Xu, G.; Song, Z.; Ding, Z.; Pang, S. Prevalence, correlates, comorbidity, and age of onset of alcohol use disorders in adult males from five provinces in China. Drug Alcohol Depend. 2017, 173, 170-177. [CrossRef] [PubMed]

13. Newman, I.M.; Ding, L.Y.; Feng, Y.H. Estimate of undergraduate university student alcohol use in China: A systematic review and meta-analysis. Arch. Public Health 2017, 75, 52. [CrossRef] [PubMed] 
14. Feng, Y.H.; Newman, I.M. Estimate of adolescent alcohol use in China: A meta-analysis. Arch. Public Health 2016, 74, 45. [CrossRef] [PubMed]

15. Taylor, A.W.; Bewick, B.M.; Makanjuola, A.B.; Qian, L.; Kirzhanova, V.V.; Alterwain, P. Context and culture associated with alcohol use amongst youth in major urban cities: A cross-country population-based survey (Ilorin Nigeria; Wuhan China; Montevideo Uruguay; and Moscow Russia). PLoS ONE 2017, 12, e0187812. [CrossRef] [PubMed]

16. World Health Organization. China (Country Profiles). In Global Status Report on Alcohol and Health. 2014. Available online: http://www.who.int/substance_abuse/publications/global_alcohol_report/profiles/chn. pdf?ua=1 (accessed on 24 October 2017).

17. Hao, W.; Young, D. Drinking patterns and problems in China. J. Subst. Use 2000, 5, 71-78. [CrossRef]

18. Hao, W.; Young, D.; Xiao, S.; Li, L.; Zhang, Y. Alcohol consumption and alcohol-related problems: Chinese experience from six area samples, 1994. Addiction 1999, 94, 1467-1476.

19. Newman, I.M. Swimming with Crocodiles: The Culture of Extreme Drinking; Martinic, M., Measham, F., Eds.; Routledge: New York, NY, USA, 2008; pp. 100-110.

20. Wang, S.; Lay, S.; Yu, H.; Shen, S. Dietary Guidelines for Chinese Residents (2016): Comments and comparisons. J. Zhejiang Univ.-Sci. B 2016, 17, 649-656. (In Chinese) [CrossRef] [PubMed]

21. De Winter, J.C.; Dodou, D. Five-point Likert items: T test versus Mann-Whitney-Wilcoxon. Pr. Assess. Res. Eval. 2010, 15, 1-12.

22. Gogol, K.; Brunner, M.; Goetz, T.; Martin, R.; Ugen, S.; Keller, U.; Fischbach, A.; Preckelf, F. “My questionnaire is too long!" The assessments of motivational-affective constructs with three-item and single-item measures. Contemp. Educ. Psychol. 2014, 39, 188-205. [CrossRef]

23. Wong, H.C.A. Assessing Young Adult Drinking Practices to Develop Alcohol Harm Prevention Strategies in Hong Kong: A Mixed Methods Approach (Order No. 3707487). Available from ProQuest Dissertations \& Theses A\&I. (1691127475). 2014. Available online: https:/ /0-search.proquest.com.library.unl.edu/docview / 1691127475? accountid=8116 (accessed on 27 October 2017).

24. Meier, P.S.; Warde, A.; Holmes, J. All drinking is not equal: How a social practice theory lens could enhance public health research on alcohol and other health behaviours. Addiction 2018, 113, 206-213. [CrossRef] [PubMed]

(C) 2018 by the authors. Licensee MDPI, Basel, Switzerland. This article is an open access article distributed under the terms and conditions of the Creative Commons Attribution (CC BY) license (http:/ / creativecommons.org/licenses/by/4.0/). 